\title{
Tetranectin Knockout Mice Develop Features of Parkinson Disease
}

\author{
Er-song Wang ${ }^{a}$ Xiao-ping Zhang ${ }^{b}$ Hui-bin Yao ${ }^{a}$ Gan Wang ${ }^{c}$ Shi-wen Chen ${ }^{c}$ \\ Wen-wei Gaoc Hai-jun Yaoc Yi-rui Sun ${ }^{d}$ Cai-hua Xic Yao-dong Jia \\ aDepartment of Neurosurgery, Jinshan Hospital, Fudan University, 'Institute of Medical Intervention \\ Engineering, Tongji University; \& Department of Interventional Radiology, Shanghai Tenth People's \\ Hospital, Tongji University, Shanghai, China, 'Department of Neurosurgery, Shanghai 6th People's \\ Hospital, Shanghai Jiaotong University, Shanghai, Shanghai Traumatic Emergency Center, dDepartment \\ of Neurosurgery, Huashan Hospital, Fudan University, Shanghai Neurosurgical Center, Shanghai, China
}

\section{Key Words}

Tetranectin • Parkinson disease $\bullet$ Motor deficits $\bullet \alpha$-synuclein; $\bullet$ Lewy bodies

\begin{abstract}
Background/Aims: Aggregation of insoluble $\alpha$-synuclein to form Lewy bodies (LBs) may contribute to the selective loss of midbrain dopaminergic neurons in Parkinson disease (PD). Lack of robust animal models has impeded elucidation of the molecular mechanisms of LB formation and other critical aspects of PD pathogenesis. Methods: We established a mouse model with targeted deletion of the plasminogen-binding protein tetranectin (TN) gene $\left(\mathrm{TN}^{--}\right)$and measured the behavioral and histopathological features of PD. Results: Aged (15to 20-month-old) TN-/-mice displayed motor deficits resembling PD symptoms, including limb rigidity and both slower ambulation (bradykinesia) and reduced rearing activity in the open field. In addition, these mice exhibited more numerous $\alpha$-synuclein-positive LB-like inclusions within the substantia nigra pars compacta (SNc) and reduced numbers of SNc dopaminergic neurons than age-matched wild type (WT) mice. These pathological changes were also accompanied by loss of dopamine terminals in the dorsal striatum. Conclusion: The $\mathrm{TN}^{-/-}$ mouse exhibits several key features of PD and so may be a valuable model for studying LB formation and testing candidate neuroprotective therapies for PD and other synucleinopathies.
\end{abstract}

E.-s. Wang and X.-p. Zhang contribute equally to the study.

Yao-dong Ji
Department of Neurosurgery, Jinshan Hospital, Fudan University 


\section{Introduction}

Parkinson disease (PD) is a neurodegenerative movement disorder characterized by resting tremor, bradykinesia, rigidity, postural instability, and gait disturbances. Nonmotor symptoms, including dysfunction of the autonomic nervous system, sensory and sleep disturbances, and neuropsychiatric symptoms are also common [1]. While PD may be diagnosed at any age, it is most common in the elderly, afflicting approximately $1 \%$ of individuals over 60 years of age and $4 \%$ above 85 years of age $[2,3]$. In this late-onset PD, typically identified in individuals over the age of 70, postural imbalance and gait impairment with accompanying rigidity and akinesia are cardinal symptoms. In contrast, early-onset PD in individuals less than 50 years of age is typified by a dominant tremor and slowly progressive motor decline $[4,5]$.

The principal neuropathological features of PD are moderate to severe dopaminergic neuron loss within the substantia nigra pars compacta $(\mathrm{SNc})$ and the presence of Lewy bodies (LBs) in surviving neurons [6]. It is thought that the combination of Lewy pathology and dopaminergic cell loss leads to striatal dopamine (DA) depletion, resulting in akinesia and other motor symptoms [7]. Diagnosis before the onset of motor deficits is critical for future preservation of function because motor symptoms do not appear until approximately $60 \%$ of dopaminergic neurons in the SNc are lost [8].

The identification of biomarkers for presymptomatic PD will allow for earlier neuroprotective treatment, possibly slowing disease progression. Our previous study suggested that tetranectin (TN), a plasminogen kringle 4 binding C-type lectin, and apoA-I may serve as potential biomarkers for PD [9]. TN was first identified as a secreted protein in 1986 and is thought to regulate proteolytic processes by binding to the ubiquitous circulating protease plasminogen $[10,11]$. It is also believed to regulate mineralization in osteogenesis as well as myogenesis during both embryonic development and muscle regeneration [12, 13]. Reduced serum TN concentration in several cancers is considered an indication of poor prognosis [14]. One proteomic study reported that TN is one of four proteins elevated in the cerebrospinal fluid (CSF) of patients with multiple sclerosis [15], although another study reported high TN concentrations $(\sim 0.5 \mathrm{mg} / \mathrm{L})$ in normal CSF [16].

Tetranectin is clearly dysregulated in several diseases. In this study, we examined whether knockout of TN might induce pathological changes and motor deficits characteristic of PD. Indeed, we found that aged $\mathrm{TN}^{-/-}$mice showed several pathological, neurochemical, and behavioral features of PD, thus establishing the $\mathrm{TN}^{-/-}$mouse as a valuable model of PD pathogenesis.

\section{Materials and Methods}

Animals

TN heterozygous mice were obtained from the Shanghai Research Center for Model Organisms (license number: SCXK (Hu) 2009-0023). Heterozygous mice were backcrossed ten generations onto the C57BL6/J background. All mice were genotyped by polymerase chain reaction (PCR) of genomic DNA isolated from tail samples at weaning. The primers used for PCR genotyping were as follows: TN, Forward: $5^{\prime}$-TTC CTG TCC CAG GTC ATT GC-3', Reverse: 5'-GAA CAG CGC CTC GTT CTC TA-3'. Thirty TN $^{-\prime-}$ and 30 wild type (WT) littermates were used for comparative analysis.

\section{Evaluation of motor dysfunction}

Twelve-month-old $\mathrm{TN}^{-/-}$and WT mice were monitored by videotape and periodic behavioral testing for 12 weeks to determine the presence, severity, and progression of motor dysfunction. We adopted the semiquantitative behavioral rating scale used widely to assess Parkinsonian phenotypes in animals [17]. The rotarod test (Columbus Instruments, Columbus, $\mathrm{OH}$ ) was performed to further assess sensorimotor coordination and balance. Mice were retrained over two consecutive days on a rotarod apparatus consisting 
of a rotating spindle $8.5 \mathrm{~cm}$ in width and $7 \mathrm{~cm}$ in diameter, requiring the animal to walk forward continuously to avoid falling. The spindle was gradually accelerated from 0 to $30 \mathrm{rpm}$ over $1 \mathrm{~min}$ and continued at $30 \mathrm{rpm}$ for an additional $2 \mathrm{~min}$. Mice were given as many trials as required to reach this performance criterion, after which they were returned to their home cage until testing the following day. Mice that did not reach this criterion were eliminated from the present study. The rotarod test was conducted at the required time points with three trials for each mouse, and the turns and average latency to fall were calculated and recorded for each group. Motor activity and posture were assessed by placing $\mathrm{TN}^{-/-}$and WT mice in a large open field allowing for unrestricted and uninterrupted movement and exploration. Rigidity was assessed by measuring passive resistance to movement of the forelimbs and tail. Tremor-like limb movements were monitored in the home cage $(28.5 \times 17.5 \times 12 \mathrm{~cm}$, AccuScan, Columbus, USA). Motor deficits were rated as absent (0), mild (1), moderate (2), marked (3), or severe (4) and scores on individual tests were averaged to yield a composite score. In addition to the rating scale, motor function was assessed using a computerized three-dimensional activity monitoring system (AccuScan Digiscan Model RXYZCM) that measures activity by the frequency of infrared beam breaks as the animals' movements traverse beams in the $\mathrm{x}, \mathrm{y}$, and $\mathrm{z}$ planes. Total distance $(\mathrm{cm})$ traveled and rearing activity was automatically determined by the beam breaks in the horizontal and vertical planes. Time spent at the spot of placement in the center of the open field was also recorded. All behavioral assessments were conducted by co-authors blind to animal genotype and treatment history. For the apomorphine test, 12-month-old $\mathrm{TN}^{-/-}$and WT mice received subcutaneous (s.c.) injection of apomorphine hydrochloride (APO) at $1.0 \mathrm{mg} / \mathrm{kg}$ and the mean composite motor function score was measured as described above.

\section{Histopathological and biochemical analyses}

Twelve-month-old mice were sacrificed by intraperitoneal (i.p.) injection of overdose pentobarbital $(60 \mathrm{mg} / \mathrm{kg})$, followed by transcardial perfusion of $100 \mathrm{ml} 0.1 \%$ heparinized normal saline $(0.9 \%)$ and then $200 \mathrm{ml}$ of $4 \%$ paraformaldehyde (PFA) in phosphate-buffered saline (PBS). Brains were removed, postfixed in 4\% PFA for 1 day, cryoprotected in 30\% sucrose for 2 days, and stored in isopentane at $-70^{\circ} \mathrm{C}$ for histochemistry. Fresh tissues were used for biochemical analysis. When brains were required for both biochemical and histopathological studies, the brains were divided mid-sagittally. One hemisphere was immediately dissected on ice and stored at $-70^{\circ} \mathrm{C}$ until required for biochemical analysis. The other hemisphere was postfixed, cryoprotected, and stored as described.

Measurement of striatal DA and DA metabolites by High-pressure Liquid Chromatography (HPLC)

WT and $\mathrm{TN}^{-/-}$mice ( $\mathrm{n}=6$ per genotype) were killed by cervical dislocation and the striatal tissue dissected out, weighed, and homogenized by sonication in ice-cold $0.4 \mathrm{M}$ perchloric acid (10\% wt/ vol) containing 3,4-dihydroxybenzylamine (DHBA) as an internal standard for subsequent HPLC. Homogenates were centrifuged at $25,000 \mathrm{~g}$ for $10 \mathrm{~min}$ at $4^{\circ} \mathrm{C}$ and supernatants were collected. DA and 3,4-dihydroxyphenylacetic acid (DOPAC) concentrations were measured by electrochemical detection using the peak height ratio and expressed in pmol analyte per mg striatal tissue (original wet weight).

\section{Western blot analysis}

Striatal tissue was isolated, weighed, and homogenized in ice-cold RIPA buffer supplemented with phosphatase and protease inhibitors. After centrifugation, supernatant proteins were quantified using the Bradford assay. Proteins were then resolved by SDS-PAGE and transferred to PVDF membranes (BioRad, Hercules, CA). The membranes were incubated with anti-DAT or anti-TH primary antibody (1:500; Abcam, Cambridge, USA), washed, and incubated in a horseradish peroxidase (HRP)-conjugated secondary antibody. Bands were visualized using ECL reagent (Pierce, Rockford, USA) and quantified by ImageQuant software. $\beta$-actin was used as a protein-loading control.

\section{Immunohistochemistry}

Mice were sacrificed for immunohistochemistry by overdose of sodium pentobarbitone and intracardially perfused with PBS followed by chilled 4\% PFA in PBS. Brains were prepared as described in Section 2.3. Fifteen-micrometer sections were cut on a cryostat, blocked in PBS containing 1\% goat serum and $0.1 \%$ Triton-X 100 , and incubated at $4^{\circ} \mathrm{C}$ overnight with anti- $\alpha$-synuclein (mouse IgG, 1:500; Chemicon, CA, USA), anti-ubiquitin (rat IgG, 1:500; Chemicon), and anti-tyrosine hydroxylase (mouse IgG, 1:500; Chemicon). After washing, a streptavidin HRP complex (1:1000; Dako, Carpinteria, CA) was applied to the sections for $1 \mathrm{~h}$. Immunolabeling was visualized using a diaminobenzidine peroxidase substrate kit 
Fig. 1. Quantification of DA neurons in the SNc neurons and estimates of DA transmission in the striatum of aged $\mathrm{TN}^{-/-}$and WT mice. (A) Stereological counts revealed a significant reduction in the number of TH-immunoreactive cells in the SNc of aged $\mathrm{TN}^{-/-}$mice. (B) DAT expression were higher in the dorsal striatum of aged $\mathrm{TN}^{-/-}$mice. DA (C) and DOPAC (D) concentrations were measured in the striatum of WT and $\mathrm{TN}^{-/-}$mice and expressed as pmol per mg of tissue. (E) Relative TH expression in striatum was analyzed by western blot. Data are the mean \pm SD. Bars represent SD. ${ }^{*} p<0.05$, ${ }^{* *} p<0.01$.
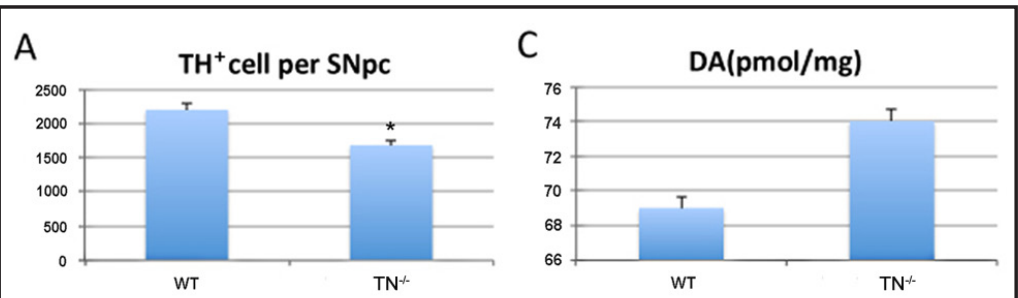

B

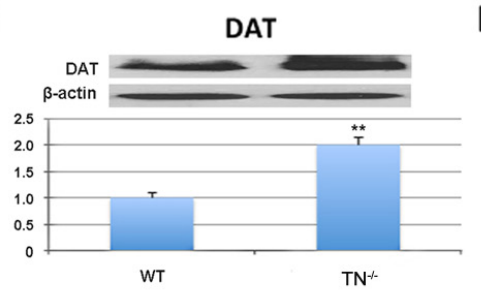

D

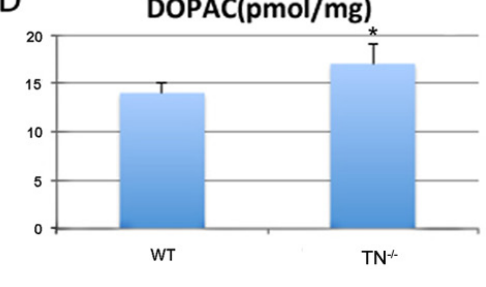

E

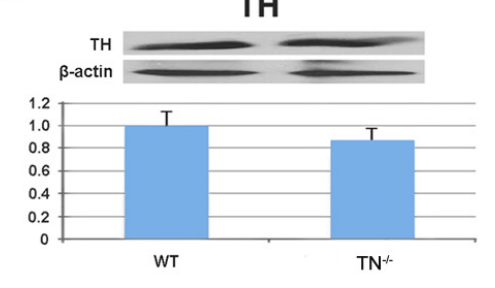

(Vector Labs, Burlingame, CA). Sections were counterstained with hematoxylin and eosin. We used the staining characteristics of dopaminergic cells to train machine-learning algorithms for identification and cell counting in each image using CellProfiler Analyst, which allowed manual classification of randomly presented nuclei and error correction of machine-classified nuclei.

\section{Gene expression analysis}

Quantitative real-time PCR (qPCR) was used for gene expression analysis of $\alpha$-synuclein. Fresh brain tissue was pooled according to genotype and RNA isolated using the Picopure RNA Extraction Kit (Arcturus, Carlsbad, USA). The RNA was reverse-transcribed using the Superscript III qPCR RT Kit (Invitrogen, Burlington, Canada) and qPCR performed using the SYBR Green qPCR SuperMix Universal (Invitrogen) on an ABI 9700 system. For insoluble $\alpha$-synuclein quantification, tissue was treated with proteinase $\mathrm{K}$ to digest the soluble proteins and then processed as described above. Primers were designed using Primer 5.0. Relative expression was measured using the $\Delta \Delta \mathrm{CT}$ method; beta-actin was used as the internal reference. Primer sequences used were as follows: $\alpha$-synuclein, Forward: 5'-TCT GGT CAA CAG CCA AAT CCT-3', Reverse: $5^{\prime}$-AAC ATT TAC CTC TAG TCA GTT GGA-3'; beta-actin, Forward: $5^{\prime}$-GAT CTT GAT CTT CAT GGT GCT AG-3', Reverse: 5'-TTG TAA CCA CCT GGG ACG ATA TGG-3'.

\section{Statistics}

Statistical analyses were conducted using SPSS 12.0 software (SPSS, Chicago, IL, USA). For normally distributed data, paired means were compared using Student's $t$ tests and multiple group means by twoway analysis of variance (ANOVA) and post hoc Tukey pair-wise comparisons. Motor function scores were compared using the nonparametric Freidman test and Dunnett's post test. A $p<0.05$ was regarded as significant. Significant changes are marked with a single asterisk in figures, irrespective of the $p$ value.

\section{Results}

Aged $\mathrm{TN}^{-/-}$mice have fewer dopaminergic SNc neurons

The number of dopaminergic neurons in the SNc, as measured by counting TH-positive cells in stained sections, was significantly lower in 12 -month-old $\mathrm{TN}^{-/-}$mice compared to 
A

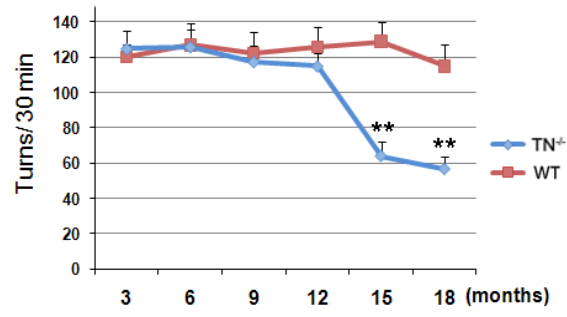

C

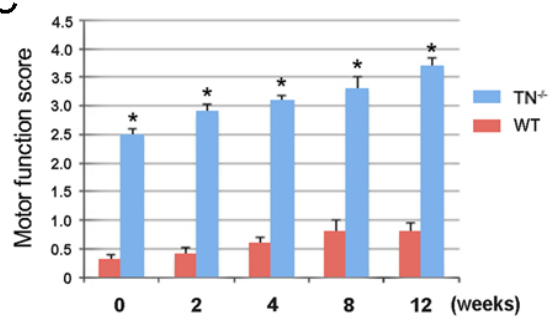

E

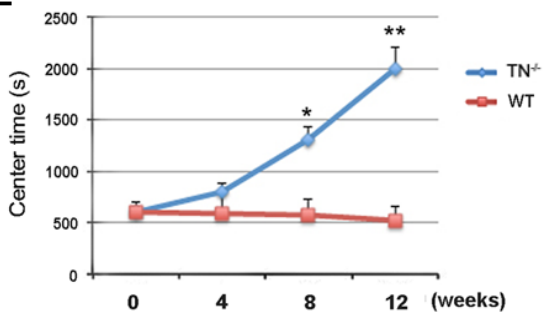

B

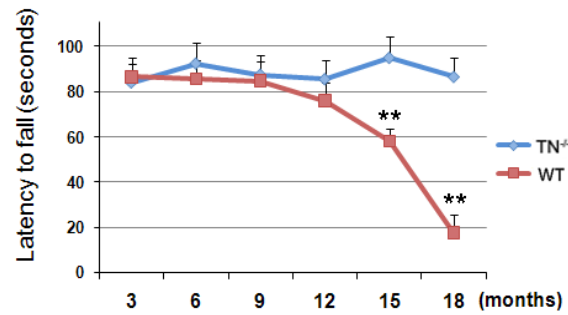

$\mathrm{D}$

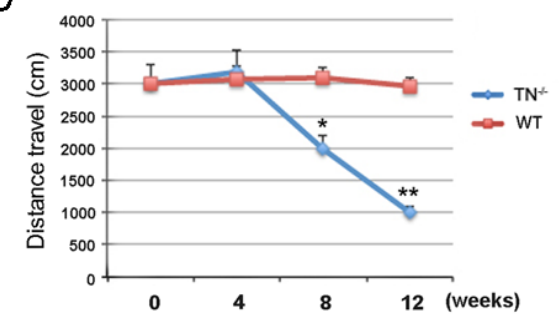

$\mathrm{F}$

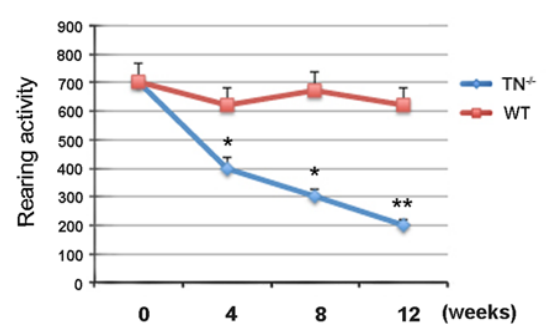

G

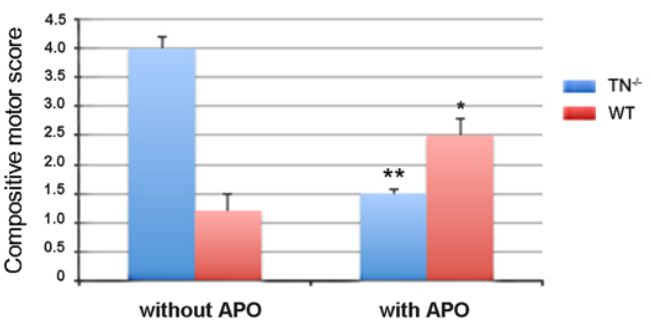

Fig. 2. Assessment of motor function in $\mathrm{TN}^{-/-}$and WT mice. There were no behavior differences between WT and $\mathrm{TN}^{-/-}$mice one year after birth. Older $\mathrm{TN}^{-/-}$mice (> 12 months old) showed slower rotation (A) and shorter latency to fall (B) than WT mice in the rotarod test. (C) Bradykinesia, rigidity, tremor-like limb movements, and abnormal posture were rated in each animal as 0 (absent), 1 (mild), 2 (moderate), 3 (marked), or 4 (severe) and averaged to yield the motor function score. Aged $\mathrm{TN}^{-/-}$mice had significantly higher scores (poorer motor function) at all times from week 0 to week 12 (starting from 12 months after birth). Motor function was assessed both manually and using a computerized three-dimensional activity monitoring system. (D) Total distance travelled in the open field. (E) Time spent at the central placement site. (F) Rearing activity as measured by Y-axis beam breaks. (G) Comparison of motor activity before and after administration of APO $(1.0 \mathrm{mg} / \mathrm{kg})$. Composite motor score includes bradykinesia, rigidity, and abnormal posture but excludes tremor-like limb movements because this behavior did not respond significantly to APO. Scores were improved by APO (mean, approximately $72 \%$ ), but motor responses were never fully restored. Data are the mean \pm SD. ${ }^{*} p<0.05,{ }^{* *} p<0.01$.

age-matched WT mice (Fig. 1A). In contrast, the content of the DAT was two-fold higher in the striatum of $\mathrm{TN}^{-/-}$mice (Fig. 1B), indicating a substantial increase in the number of striatal DA terminals per SNc neuron. Although DA and TH contents were similar in the striatum of aged 


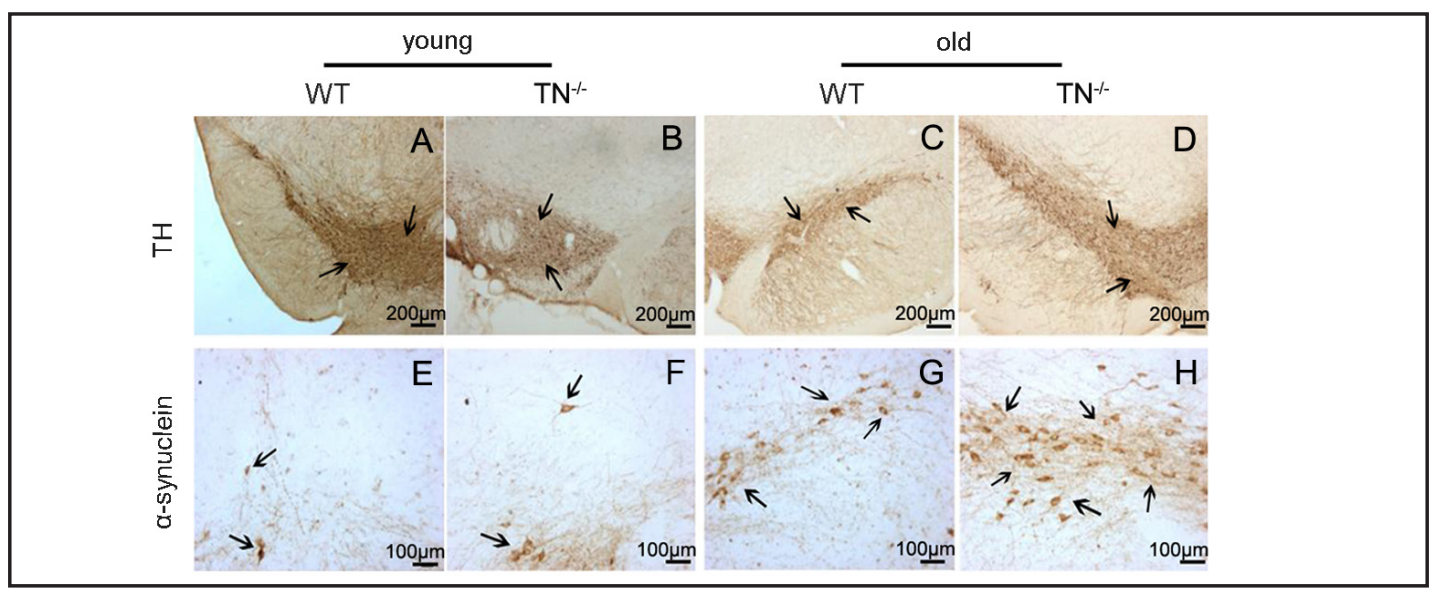

Fig. 3. TH and $\alpha$-synuclein immunoreactivity in the midbrain of young $(<6$ months old) and old $(>18$ months old) wild type and $\mathrm{TN}^{-/-}$mice (A-D) Photomicrographs of TH immunoreactivity in the midbrain of young WT (A), young $\mathrm{TN}^{-/-}$(B), old WT (C), and old $\mathrm{TN}^{-/-}$mice (D). (E-H) $\alpha$-synuclein staining in adjacent midbrain sections from young WT (E), young $\mathrm{TN}^{-/-}(\mathrm{F})$, old WT (G), and old $\mathrm{TN}^{-/-}$mice (H) demonstrating the increased $\alpha$-synuclein deposition in TH-positive (dopaminergic) nigral neurons of aging $\mathrm{TN}^{-/-}$mice.

WT and $\mathrm{TN}^{-/-}$mice (Fig. 1C and E), DOPAC levels were significantly elevated in $\mathrm{TN}^{-/-}$mice (Fig. 1D). Taken together, these results imply that despite a reduction in the number of SNc neurons, there is a compensatory increase in striatal dopaminergic terminals in $\mathrm{TN}^{-/-}$mice.

\section{Development of progressive Parkinsonism}

Behavioral analyses were performed by an observer blind to genotype and treatment history. There were no behavior differences between young WT and $\mathrm{TN}^{-/-}$mice. However, $\mathrm{TN}^{-/-}$mice $>12$ months old showed slower rotation (Fig. $2 \mathrm{~A}$ ) and shorter latency to fall in the rotarod test (Fig. 2B) than age-matched WT mice. At approximately 12 months, all $\mathrm{TN}^{-/-}$animals developed mild bradykinesia (Fig. 2C-F) as measured by lower ambulation and rearing in the open field and motor function scores. During the next 12 weeks, motor dysfunction gradually deteriorated to severe bradykinesia accompanied by moderate to severe limb rigidity and abnormal posture. $\mathrm{TN}^{-/-}$mice also displayed intermittent episodes (up to $3 \mathrm{~s}$ ) of tremor-like movements in one or both forelimbs when rearing on hindlimbs. However, it is not possible to state with certainty whether this was a resting or postural tremor. Administration of APO (1.0 mg/kg, s.c.) improved bradykinesia, rigidity, and posture in $\mathrm{TN}^{-/-}$mice as measured by the mean composite motor function score (Fig. 2G). Similarly, L-3,4-dihydroxyphenylalanine (L-DOPA) methyl ester (50 mg/kg, i.p.) improved motor function (data not shown). No spontaneous behavioral recovery was observed during the 12 weeks of observation.

\section{$\alpha$-synuclein is redistributed in SNc neurons of aged mice}

The distribution of $\alpha$-synuclein in the brain was examined in young and old WT and $\mathrm{TN}^{-/-}$mice by immunohistochemistry (Fig. 3). The location of $\alpha$-synuclein-expressing cells was confirmed by comparing adjacent sections stained for $\alpha$-synuclein or TH (Fig. 3). We observed an increase in nigral $\alpha$-synuclein immunoreactivity in older mice $(>18$ months old) compared to younger mice ( $<6$ months old) in both genotypes, but the difference was larger in $\mathrm{TN}^{-/-}$mice (compare Fig. $3 \mathrm{E}$ to $3 \mathrm{H}$ ), suggesting accelerated formation of inclusions with age.

\section{Subcellular localization of $\alpha$-synuclein}

We also compared the subcellular localization of $\alpha$-synuclein immunoreactivity in the SNc of young and old mice of each genotype. Three subcellular distribution patterns were 


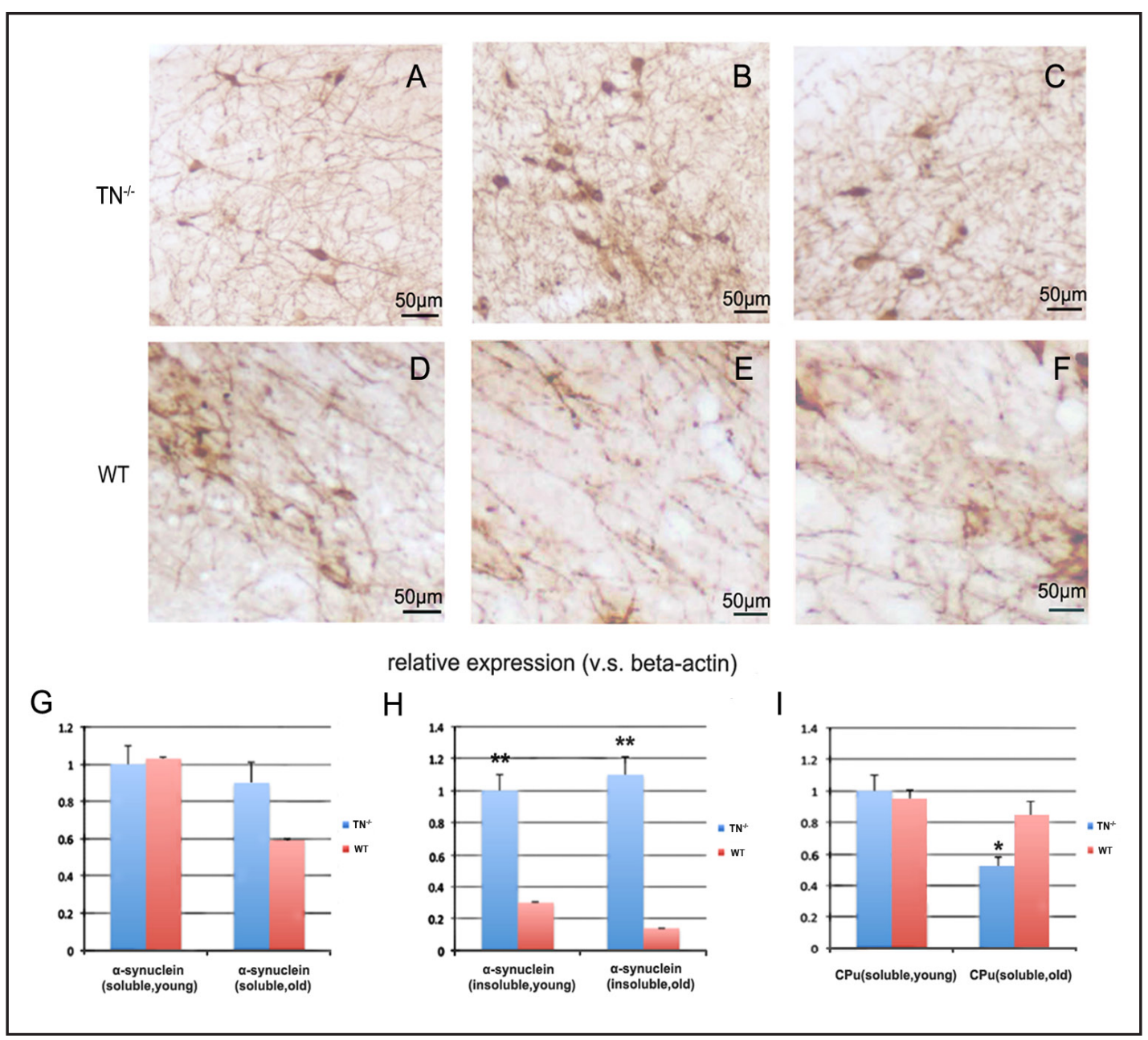

Fig. 4. Changes in the subcellular distribution of $\alpha$-synuclein with age in SNc neurons of WT and $\mathrm{TN}^{-/-}$mice (A-F) Three staining patterns were noted, cytoplasmic (A and D), nuclear (B and E), and combined cytoplasmic and nuclear staining ( $\mathrm{C}$ and F). The intensity of $\alpha$-synuclein staining was elevated in older animals of both genotypes for all three subcellular distribution patterns, but staining was more intense in aged $\mathrm{TN}^{-/-}$mice (A-C) compared to aged WT mice (D-F). (G-I) Redistribution of $\alpha$-synuclein was determined by qRT-PCR. Relative expression of beta-actin was used for normalization. (G) There were no age-dependent changes in soluble $\alpha$-synuclein. (H) The expression of insoluble $\alpha$-synuclein was higher in both young and aged $\mathrm{TN}^{-/-}$mice compared to age-matched WT mice. (I) Conversely, soluble $\alpha$-synuclein was reduced in the caudate putamen/striatum $(\mathrm{CPu})$ of aged $\mathrm{TN}^{-/-}$mice. Data are the mean $\pm \mathrm{SD}$. ${ }^{*} p<0.05,{ }^{* *} p<0.01$.

recognized by immunostaining: cytoplasm only (Fig 4A and D), nucleus only (Fig 4B and E), and staining in both compartments (Fig. 4C and F). Regardless of the location, immunoreactivity was more intense in old $\mathrm{TN}^{-/-}$mice (Fig. 4A-C) than age-matched WT mice (Fig. 4D-F). qPCR was performed to measure soluble and aggregated insoluble $\alpha$-synuclein in nigral and striatal tissue homogenates from young and old WT and $\mathrm{TN}^{-/-}$mice (Fig. 4G-I). The amount of soluble $\alpha$-synuclein in the SNc did not change with age in mice of either genotype (Fig. $4 \mathrm{G}$ ). However, expression of insoluble $\alpha$-synuclein was significantly higher in aged mice (Fig. $4 \mathrm{H}$ ) and there was a trend for elevated insoluble $\alpha$-synuclein in the SNc of both young and old $\mathrm{TN}^{-/-}$mice compared to age-matched WT littermates. In contrast, striatal $\alpha$-synuclein levels were significantly lower in aged $\mathrm{TN}^{-/-}$mice (Fig. 4I). These findings, together with the immunohistological data, suggest the loss of soluble $\alpha$-synuclein in dopaminergic terminals within the striatum and accumulation of insoluble $\alpha$-synuclein in the soma. 


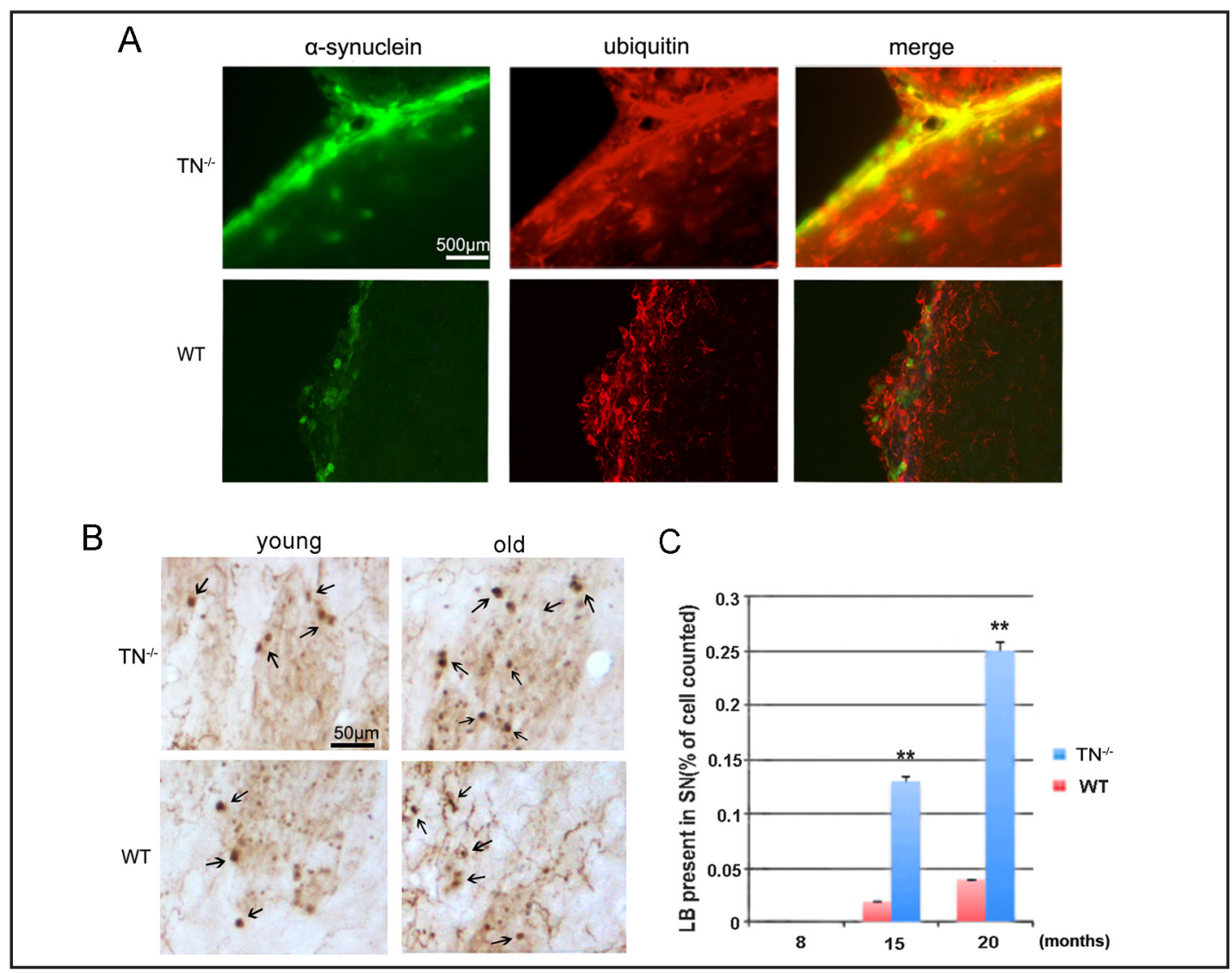

Fig. 5. $\mathrm{TN}^{-/-}$mice develop more LB-like inclusions than WT mice. Fluorescence immunohistochemistry revealed colocalization of ubiquitin and $\alpha$-synuclein in SNc neurons of aged $\mathrm{TN}^{-/-}$and WT mice. (A) Sections were stained with $\alpha$-synuclein (green) and ubiquitin (red). The merged image indicates the degree of overlap (yellow). These $\alpha$-synuclein/ubiquitin-immunoreactive aggregates displaced the nucleus in many neurons. (B) $\alpha$-synuclein-immunoreactive LB-like inclusions (arrows) in $\mathrm{WT}$ and $\mathrm{TN}^{-/-}$mice were also examined by immunostaining with HPR visualization. An example from a 15-month-old $\mathrm{TN}^{-/-}$mouse (younger TN) showing the tendency for aggregates to have a dense eosinophilic core with lower immunoreactivity and to displace the nucleus. Lewy neurites were also occasionally observed in 20-month-old WT mice (old WT). (C) Stereological counts of the fraction of SNc neurons containing LBs in WT and $\mathrm{TN}^{-/-}$mice. Data are the mean \pm SD. ${ }^{* *} p<0.01$.

\section{More LB-like inclusions form in aged $\mathrm{TN}^{-/-}$mice}

If the redistribution of $\alpha$-synuclein observed in aged mice is a pathogenic process analogous to that occurring in PD, we would also expect to see ubiquitinated inclusions and the occurrence of LBs. Nigral neurons in aged mice of both genotypes exhibited $\alpha$-synuclein inclusions that were also positive for ubiquitin (Fig. 5A). Immunoreactivity was frequently punctate rather than diffuse, suggesting that microinclusions were being formed. In addition to increased diffuse and punctate staining, we also observed large dense $\alpha$-synucleinpositive inclusions within the cell bodies of SNc neurons in aged WT and $\mathrm{TN}^{-/-}$mice (Fig. 5B). These positive inclusions were morphologically and histologically similar to the LBs seen in human PD brains. The number of cells in the SNc containing LB-like inclusions was counted and expressed as a percentage of the total number of neurons in the SNc (Fig. 5C). $\alpha$-synuclein inclusions were not seen in 8-month-old mice of either genotype. A significantly higher percentage of SNc neurons in older $\mathrm{TN}^{-/-}$mice exhibited inclusions compared to agematched WT mice. The distribution of inclusions was not homogeneous, with inclusions commonly observed in clusters. 


\section{Discussion}

Current available treatments can manage the symptoms of PD, but there are no effective methods to halt disease progression $[1,6]$. Clinical treatments are mainly limited to the DA precursor L-DOPA and inhibitors of DA metabolism, which supplement reduced striatal DA [18], and deep-brain stimulation of the basal ganglia, which can alleviate tremor [19]. The development of treatments that actually arrest PD progression requires a more detailed understanding of the molecular mechanisms responsible for LB formation and other early pathogenic events that precede the loss of dopaminergic neurons.

Neuronal inclusions containing $\alpha$-synuclein are a pathological hallmark of PD and may be critical in disease pathogenesis and progression. However, little is known of the molecular mechanisms leading to the aggregation of $\alpha$-synuclein and formation of LBs in the SNc and elsewhere in the brain. Here we demonstrate that loss of the plasminogen-binding protein $\mathrm{TN}$ in mice accelerates the age-dependent formation of LBs and results in motor deficits similar to those of PD. The histopathological and behavioral characteristics of these mice may prove valuable for studies aimed at elucidating the genesis of LBs and their role in neurodegeneration.

Tetranectin is thought to play a prominent role in the regulation of tissue remodeling, osteogenesis, myogenesis, wound repair and regeneration [12, 13, 20, 21]. Dietzmann et al. demonstrated that $\mathrm{TN}$ was overexpressed immunohistochemically in reactive astrocytes, microglia, and endothelial cells around the lesion zone in the brain following circulatory arrest, suggesting that overexpression of TN may impede tissue repair [22]. Our previous study suggested TN may serve as a potential biomarker for PD [9]. In the current study, we found that TN knockout induced $\alpha$-synucleinopathy and Parkinsonian motor symptoms, possibly by interfering with the clearance of $\alpha$-synuclein or accelerating aggregation. We have thus shown that reduced TN levels control the formation of the insoluble toxic $\alpha$-synuclein assemblies in mouse brain associated with the degeneration of nigral dopaminergic neurons.

The formation of $\alpha$-synuclein assemblies inhibits the lysosomal activity of glucocerebrosidase in neurons of the idiopathic PD brain, resulting in a self-propagating positive-feedback cycle of $\alpha$-synuclein accumulation that eventually reaches a pathogenic threshold [23]. This lysosomal proteolytic dysfunction (LSD) is one of the common mechanisms underlying the $\alpha$-synuclein pathology shared by various disorders. Dysfunction of the ubiquitin-proteasome system also underlies many of these $\alpha$-synucleinopathies [24, 25]. The parkin gene associated with a recessive familial early-onset PD encodes the E3 ubiquitin ligase involved in ubiquitination of misfolded glucocerebrosidase in dopaminergic neurons. The absence of normal parkin leads to improper degradation of $\alpha$-synuclein and other E3 substrates, resulting in protein accumulation. The accumulation of ubiquitinated protein inclusions resembling LBs strongly suggests dysregulation of these protein degradation pathways in $\mathrm{TN}^{-/-}$mice.

APO, a potent, non-selective, direct-acting dopamine D1 and D2 receptor agonist, was the first dopamine receptor agonist used to treat Parkinson disease, and so had effect on motor deficits in PD $[26,27]$. In our study, $\mathrm{TN}^{-/-}$mice displayed several motor deficits, such as mild to severe bradykinesia, moderate to severe limb rigidity and abnormal posture compared to WT mice. However, it is not possible to state with certainty whether this was a resting or postural tremor. Administration of APO improved motor scores in $\mathrm{TN}^{-/-}$mice, while aggravated motor dysfunction in WT mice with elevated composite motor function score, which confirmed that $\mathrm{TN}^{-/-}$mice could mimic the symptoms of motor deficits in PD. This study also examined whether TN knockout altered the concentration of DA, DA metabolites, and $\alpha$-synuclein in the striatum. We confirmed that DA was elevated in the striatum of aged $\mathrm{TN}^{-/-}$mice, while soluble $\alpha$-synuclein was reduced in dopaminergic terminals. The decrease in soluble $\alpha$-synuclein occurred concomitantly with an increase in insoluble $\alpha$-synuclein in 
the soma. Insoluble $\alpha$-synuclein is prone to aggregation and indeed formed LB-like inclusions in mouse SNc neurons. These mouse LBs share many features with those observed in PD brains.

Ideally, a robust animal model of PD will exhibit progressive LB formation, loss of striatal dopamine terminals, loss of dopaminergic neurons in the SNc, and behavioral endophenotypes of PD, including motor and other behavioral symptoms [18, 19]. We suggest that many of the pathogenic processes leading to $\mathrm{PD}$ are present in $\mathrm{TN}^{-/-}$mice, such as inclusion formation and increased striatal DA and DAT, which are signs of a compensatory response and model the preclinical disease. These features are not consistently present in other animal models, however, underscore the value of $\mathrm{TN}^{-/-}$mice for future studies. The milder neurological and behavioral manifestation in mice may reflect the shorter life span of these animals.

The $\mathrm{TN}^{-/-}$mouse may provide a new model of synucleinopathy suitable for studying the early pathogenic processes in PD and other neurodegenerative diseases. Oxidative stress due to DA catabolism and the extensive plasticity of DA terminals may increase the vulnerability of the human SNc to $\alpha$-synuclein accumulation, LB formation, and neurodegeneration. Knockout of TN may exacerbate these mechanisms by increasing the number of terminal arbors per neuron. However, it remains unknown whether TN knockout also leads to other diseases or dysfunction of other organs. Future studies are needed to establish the relative contribution of each of these pathogenic processes to SNc neuron degeneration and determine which are most amenable to clinical intervention.

In conclusion, we show that tetranectin knockout can induce a progressive neurodegenerative disorder in mice that closely recapitulates the features of PD. These studies raise the possibility that a hypofunctional TN gene contributes to the development of the illness in susceptible individuals. In addition, this animal model of progressive PD could provide new insights into mechanisms involved in the neurodegenerative process, and may be useful for developing neuroprotective therapies.

\section{Acknowledgements}

This work was supported by grants from Shanghai Municipal Commission for Science and Technology (No. 10140903500 and 124119a1900), Shanghai Jiaotong University (No. YG2010MS40) and Shanghai Municipal Public Health Bureau (No.20124339)

\section{Disclosure Statement}

The authors declare no conflict of interest.

\section{References}

1 Park A, Stacy M: Non-motor symptoms in parkinson's disease. J Neurol 2009;256:S293-298.

2 de Lau LM, Breteler MM: Epidemiology of parkinson's disease. Lancet Neurol 2006;5:525-535.

- Van Den Eeden SK, Tanner CM, Bernstein AL, Fross RD, Leimpeter A, Bloch DA, Nelson LM: Incidence of parkinson's disease: Variation by age, gender, and race/ethnicity. Am J Epidemiol 2003;157:1015-1022.

-4 Liu CW, Giasson BI, Lewis KA, Lee VM, Demartino GN, Thomas PJ: A precipitating role for truncated alphasynuclein and the proteasome in alpha-synuclein aggregation: Implications for pathogenesis of parkinson disease. J Biol Chem 2005;280:22670-22678.

5 Selikhova M, Williams DR, Kempster PA, Holton JL, Revesz T, Lees AJ: A clinico-pathological study of subtypes in parkinson's disease. Brain 2009;132:2947-2957. 
Wang et al.: Tetranectin Knockout Mice Develop Features of Parkinson Disease

6 Dickson DW, Braak H, Duda JE, Duyckaerts C, Gasser T, Halliday GM, Hardy J, Leverenz JB, Del Tredici K, Wszolek ZK, Litvan I: Neuropathological assessment of parkinson's disease: Refining the diagnostic criteria. Lancet Neurol 2009;8:1150-1157.

-7 Obeso JA, Rodriguez-Oroz MC, Goetz CG, Marin C, Kordower JH, Rodriguez M, Hirsch EC, Farrer M, Schapira AH, Halliday G: Missing pieces in the parkinson's disease puzzle. Nat Med;16:653-661.

-8 Fearnley JM, Lees AJ: Ageing and parkinson's disease: Substantia nigra regional selectivity. Brain 1991;114 (Pt 5):2283-2301.

-9 Wang ES, Sun Y, Guo JG, Gao X, Hu JW, Zhou L, Hu J, Jiang CC: Tetranectin and apolipoprotein a-i in cerebrospinal fluid as potential biomarkers for parkinson's disease. Acta Neurol Scand;122:350-359.

$\checkmark 10$ Clemmensen I, Petersen LC, Kluft C: Purification and characterization of a novel, oligomeric, plasminogen kringle 4 binding protein from human plasma: Tetranectin. Eur J Biochem 1986;156:327-333.

11 Obrist P, Spizzo G, Ensinger C, Fong D, Brunhuber T, Schafer G, Varga M, Margreiter R, Amberger A, Gastl G, Christiansen M: Aberrant tetranectin expression in human breast carcinomas as a predictor of survival. J Clin Pathol 2004;57:417-421.

12 Wewer UM, Iba K, Durkin ME, Nielsen FC, Loechel F, Gilpin BJ, Kuang W, Engvall E, Albrechtsen R: Tetranectin is a novel marker for myogenesis during embryonic development, muscle regeneration, and muscle cell differentiation in vitro. Dev Biol 1998;200:247-259.

13 Wewer UM, Ibaraki K, Schjorring P, Durkin ME, Young MF, Albrechtsen R: A potential role for tetranectin in mineralization during osteogenesis. J Cell Biol 1994;127:1767-1775.

14 Hogdall CK, Soletormos G, Nielsen D, Norgaard-Pedersen B, Dombernowsky P, Clemmensen I: Prognostic value of serum tetranectin in patients with metastatic breast cancer. Acta Oncol 1993;32:631-636.

15 Liu S, Bai S, Qin Z, Yang Y, Cui Y, Qin Y: Quantitative proteomic analysis of the cerebrospinal fluid of patients with multiple sclerosis. J Cell Mol Med 2009;13:1586-1603.

-16 Stoevring B, Jaliashvili I, Thougaard AV, Ensinger C, Hogdall CK, Rasmussen LS, Sellebjerg F, Christiansen M: Tetranectin in cerebrospinal fluid of patients with multiple sclerosis. Scand J Clin Lab Invest 2006;66:577583.

17 Imbert C, Bezard E, Guitraud S, Boraud T, Gross CE: Comparison of eight clinical rating scales used for the assessment of mptp-induced parkinsonism in the macaque monkey. J Neurosci Methods 2000;96:71-76.

18 Golembiowska K, Dziubina A, Kowalska M, Kaminska K: Effect of adenosine a(2a) receptor antagonists on l-dopa-induced hydroxyl radical formation in rat striatum. Neurotox Res 2009;15:155-166.

19 Seif C, Junemann KP, Braun PM: Deafferentation of the urinary bladder and implantation of a sacral anterior root stimulator (sars) for treatment of the neurogenic bladder in paraplegic patients. Biomed Tech (Berl) 2004;49:88-92.

20 Iba K, Hatakeyama N, Kojima T, Murata M, Matsumura T, Wewer UM, Wada T, Sawada N, Yamashita T: Impaired cutaneous wound healing in mice lacking tetranectin. Wound Repair Regen 2009;17:108-112.

21 Iba K, Sawada N, Chiba H, Wewer UM, Ishii S, Mori M: Transforming growth factor-beta 1 downregulates dexamethasone-induced tetranectin gene expression during the in vitro mineralization of the human osteoblastic cell line sv-hfo. FEBS Lett 1995;373:1-4.

22 Dietzmann K, von Bossanyi P, Krause D, Wittig H, Mawrin C, Kirches E: Expression of the plasminogen activator system and the inhibitors pai-1 and pai-2 in posttraumatic lesions of the cns and brain injuries following dramatic circulatory arrests: An immunohistochemical study. Pathol Res Pract 2000;196:15-21.

-23 Devine MJ, Gwinn K, Singleton A, Hardy J: Parkinson's disease and alpha-synuclein expression. Mov Disord 2011;26:2160-2168.

-24 Fishman-Jacob T, Reznichenko L, Youdim MB, Mandel SA: A sporadic parkinson disease model via silencing of the ubiquitin-proteasome/e3 ligase component skp1a. J Biol Chem 2009;284:32835-32845.

25 Fornai F, Schluter OM, Lenzi P, Gesi M, Ruffoli R, Ferrucci M, Lazzeri G, Busceti CL, Pontarelli F, Battaglia G, Pellegrini A, Nicoletti F, Ruggieri S, Paparelli A, Sudhof TC: Parkinson-like syndrome induced by continuous mptp infusion: Convergent roles of the ubiquitin-proteasome system and alpha-synuclein. Proc Natl Acad Sci U S A 2005;102:3413-3418.

26 Deleu D, Hanssens Y, Northway MG: Subcutaneous apomorphine: An evidence-based review of its use in parkinson's disease. Drugs Aging 2004;21:687-709.

27 Frankel JP, Lees AJ, Kempster PA, Stern GM: Subcutaneous apomorphine in the treatment of parkinson's disease. J Neurol Neurosurg Psychiatry 1990;53:96-101. 